\title{
Germanica
}

\section{Aux sources du conte : le mythe "Circé et Ulysse » de Franz Fühmann}

Von Märchen zum Mythos Franz Fühmanns Ballett «Kirke und Odysseus»

\section{Geneviève Cimaz-Martineau}

\section{(2) OpenEdition}

\section{Journals}

Édition électronique

URL : http://journals.openedition.org/germanica/1341

DOI : 10.4000/germanica. 1341

ISSN : 2107-0784

Éditeur

Université de Lille

Édition imprimée

Date de publication : 1 janvier 1992

Pagination : $59-70$

ISSN : 0984-2632

Référence électronique

Geneviève Cimaz-Martineau, «Aux sources du conte : le mythe « Circé et Ulysse » de Franz Fühmann », Germanica [En ligne], 11 | 1992, mis en ligne le 07 février 2014, consulté le 06 octobre 2020. URL : http://journals.openedition.org/germanica/1341 ; DOI : https://doi.org/10.4000/germanica.1341

Ce document a été généré automatiquement le 6 octobre 2020.

(c) Tous droits réservés 


\title{
Aux sources du conte : le mythe «Circé et Ulysse » de Franz Fühmann
}

\author{
Von Märchen zum Mythos Franz Fühmanns Ballett «Kirke und Odysseus»
}

\author{
Geneviève Cimaz-Martineau
}

1 Lorsque Franz Fühmann reprend l'histoire de Circé et d'Ulysse au début des années $1980^{1}$, le retour à la mythologie est à l'ordre du jour en R.D.A. Certes, Ulysse et Circé ont continué à hanter les imaginations et à inspirer les écrivains au XXe siècle. Il suffit pour s'en convaincre de consulter l'ouvrage d'Elisabeth Frenzel, Stoffe der Weltliteratur ${ }^{2}$ : à côté de Joyce, dont le roman est cité par Fühmann, nous trouvons d'Annunzio, Hauptmann, Giono, Giraudoux, mais les adaptations tirées de la mythologie se multiplient tout particulièrement autour de 1980 en R.D.A. En 1981 St. Schütz consacre une pièce au retour d'Ulysse (Odysseus' Heimkehr). En 1982 J. Berg écrit une Iphigenie et Peter Hacks une Pandora. En 1983, un an avant la parution du texte de Fühmann, Christa Wolf publie sa célèbre Cassandre, J. Berg porte à la scène l'histoire de Clytemnestre et Heiner Müller celle des Argonautes. Ce choix a de multiples raisons, dont les plus évidentes sont la volonté de se rattacher à la grande tradition littéraire et de parler de problèmes actuels par le truchement des mythes éternels, pour échapper à une réalité banale et contraignante et à la censure, comme Sartre l'avait fait dans Les Mouches.

2 Lorsque Fühmann aborde le sujet de Circé et d'Ulysse il a déjà écrit un Prométhée ${ }^{3}$. Circé et Ulysse intéressent Fühmann car ce thème est un point de rencontre entre le conte et le mythe. Gero von Wilpert considère en effet l'épisode de Circé comme un motif de conte au milieu de l'épopée qu'est l'Odyssée ${ }^{4}$, d'autre part il définit le mythe comme une histoire où se rencontrent dieux et héros et l'épisode de Circé et d'Ulysse répond également à cette définition. Fühmann a médité et écrit sur ces deux notions du conte et du mythe, d'abord sous formes d'aphorismes dans son journal de voyage en Hongrie 22 Tage oder die Hälfte des Lebens ${ }^{5}$, puis, deux ans plus tard, en 1974, il reprend le sujet des rapports du mythe et du conte dans une conférence intitulée Das mythische Element in der Literatur ${ }^{6}$. Nous verrons ce qu'il entend par ces deux termes, puis, en fonction de 
cette définition, dans quelle mesure il réutilise les éléments de la tradition homérique concernant Circé et Ulysse et quelles modifications il apporte dans l'adaptation qu'il fait de cette histoire. Nous nous efforcerons de montrer comment il sollicite les motifs de conte pour les rapprocher du mythe.

Fühmann choisit la forme du ballet qu'il projette de créer en collaboration avec une musicienne, Ruth Zechlin, dont il a fait la connaissance grâce à son ami le sculpteur Wieland Förster, au cours d'une séance de l'Académie à Rostock en mars 1981'. Ruth Zechlin renonce bientôt à composer la musique, faute de commanditaire, mais Fühmann publie le texte qui devait être l'argument du ballet. Il se sert des moyens spécifiques de cet art pour souligner ses intentions. De plus, le texte est accompagné de dessins de Wieland Förster qui ne sont pas conçus à proprement parler comme des illustrations. Förster les a intitulés «Couples d'amants» (Liebespaare) et ils entretiennent par ce motif même un rapport étroit avec Circé et Ulysse ${ }^{8}$.

Avant d'aborder dans son journal de voyage en Hongrie les rapports du mythe et du conte, Fühmann a repris en 1962, sous forme de poèmes, les contes de Grimm9 ${ }^{9}$. En 1966, il a publié des récits dont le titre König Ödipus fait allusion au mythe d'œEdipe. Mythes et contes l'ont également inspiré, mais lorsqu'il compare les deux notions, ce qui nous frappe est la dévalorisation du conte par rapport au mythe. Dans sa conférence, il prend un exemple qui illustre ce qui les distingue et il exprime cette différence dans le journal sous forme d'aphorismes. Soit un même motif : celui de la jeune fille poursuivie. Dans le conte elle est en butte aux attaques d'un méchant magicien, des griffes duquel va la sauver un bon prince qu'elle épouse. Dans le mythe, elle est séduite par un dieu dont elle a une fille qui venge, par les méfaits que sa beauté produit sur les hommes, l'offense faite à sa mère. Quelles conclusions Fühmann a-t-il tirées de ces deux schémas de récit? Le conte tout d'abord entre dans un système de références morales. Le choix y est simple entre le sorcier et le prince, le héros triomphe. Le mythe en revanche fait ressortir les contradictions à l'intérieur d'un même personnage, ainsi le dieu est-il à la fois l'amant et le séducteur qui abandonne sa victime. Or Fühmann montre que les contradictions sont au cœur de toutes les expériences fondamentales et en particulier de l'amour. C'est pourquoi les règles du jeu sont, selon lui, beaucoup plus subtiles dans le mythe que dans le conte. Les mythes " communiquent une expérience", apprennent à vivre, alors que les contes ont pour fonction de nous « consoler » et de nous " faire rêver ». Le conte est une dégradation du mythe, il ne fait que suggérer les "abîmes » qu'ouvre le mythe. Celui-ci, processus en cours d'évolution, est "multidimensionnel ", les contes ne sont que des "vestiges $»^{10}$ de mythes, des $"$ mythes engloutis $»^{11}$. C'est assez dire que Fühmann privilégie le mythe par rapport au conte et lorsqu'il reprend l'histoire de Circé et d'Ulysse, il s'efforce de gommer certains éléments de merveilleux pour mettre l'accent sur ce qui oriente cette histoire vers le mythe. Comment opère-til ?

5 Il reprend l'essentiel du récit d'Homère tel que nous le trouvons au chant $\mathrm{X}$ de l'Odyssée : pendant le voyage qui conduit Ulysse de Troie à Ithaque, il aborde sur l'île d'Aiaié, l'île de la déesse magicienne Circé. Il n'a plus qu'un bateau et quelques compagnons, parmi lesquels Euryloque et Elpénor. Circé fait perdre aux premiers compagnons d'Ulysse qui s'aventurent près de son palais leur forme humaine. Un des 
compagnons réussit à échapper à la métamorphose et revient chercher Ulysse au bateau. Celui-ci, protégé par une divinité de l'olympe, grâce à une plante, le moly ou pourpier de mer, parvient à résister aux maléfices de Circé. La déesse magicienne, ayant reconnu en Ulysse un être exceptionnel et un partenaire digne d'elle, l'invite à partager sa couche et lui accorde la faveur de retransformer ses compagnons en hommes. Ulysse demande alors à Circé de le laisser rentrer à Ithaque. Elle accepte. Athéna protège Ulysse qui finit par quitter l'île d'Aiaié, non sans avoir perdu son compagnon Elpénor qui meurt avant le départ.

6 Pourquoi a-t-on pu parler de conte à propos de cet épisode de l'Odyssée ? Si l'on examine les éléments de ce récit, on constate qu'il répond à ce que Vladimir Propp dans La Morphologie du conte ${ }^{12}$ considère comme une séquence de conte : il y a au départ un méfait, la métamorphose des compagnons d'Ulysse; le héros rencontre un être surnaturel, secourable, qui lui remet un auxiliaire magique. Cet auxiliaire doit lui permettre d'affronter la magicienne et d'obtenir l'abolition du méfait, en l'occurrence le retour des compagnons à la forme humaine. Le récit se termine par le triomphe du héros. Mais c'est moins le conte qui retient l'attention de Fühmann que le mythe, la rencontre des dieux et du héros. Ce mythe, il considère que pour être fidèle à son esprit, il faut savoir lui être, sur certains points, infidèle («Die Treue zum Mythos erfordert Untreue gegenüber allen seinen vorhandenen Fassungen $\left.»^{13}\right)$.

7 Une pure répétition du mythe homérique à notre époque ne présente pas d'intérêt et dans son ballet Fühmann apporte trois modifications importantes. Dans les trois cas, si nous tenons compte des caractéristiques qu'il a énoncées pour le conte et pour le mythe, il s'éloigne du conte pour atteindre au mythe et utilise les moyens spécifiques du ballet pour souligner cette orientation. Dans son journal de voyage Fühmann note que passer du conte au mythe, c'est passer à la vie toute entière, à l'homme dans sa totalité, à la réalité dialectique ${ }^{14}$. C'est dans ce sens en effet qu'il va traiter la métamorphose des compagnons d'Ulysse, l'intervention divine qui lui permet de résister aux maléfices de Circé et en dernier lieu la séparation d'Ulysse et de Circé. Ce chemin du conte au mythe il l'a déjà parcouru dans son Prométhée («Anfangs wollte ich den Prometheusstoff als Märchen erzählen... Ich mußte den Schritt zum Mythos tun» ${ }^{15)}$.

8 Chez Homère, Circé métamorphose les compagnons d'Ulysse en pourceaux. Fühmann puise à d'autres sources, d'abord chez Ovide qui insiste sur le processus même de la métamorphose $\mathrm{e}^{16}$, et surtout chez Apollonios de Rhodes, auquel il se réfère expressément et qui dans Les Argonautiques ${ }^{17}$ décrit ainsi le terme de la métamorphose : « Des bêtes qui n'avaient l'aspect, faute d'un corps homogène, ni de bêtes sauvages, ni non plus d'hommes, mais qui étaient faits de membres mélangés des uns et des autres ». Fühmann choisit de métamorphoser les compagnons d'Ulysse en lémures, qui dans l'Antiquité étaient des spectres et qu'il présente comme des êtres grossiers, voués à un travail mécanique et surveillés par les nymphes, ils sont massifs, courbés, certains marchent à quatre pattes. Ils ne regardent ni le ciel, ni les autres ; leurs bras touchent presque le sol, leur peau est tannée comme un cuir. Ils ont l'apparence d'êtres asexués. Lorsqu'ils font mine de se révolter, Circé leur jette de la viande et tout rentre dans l'ordre, mais Euryloque, le compagnon d'Ulysse, souffre de la métamorphose et tente d'attirer l'attention d'Ulysse pour qu'il le délivre. Ces êtres, soumis à un travail répétitif et harassant évoquent, comme le précise Fühmann (p. 24) les détenus d'un camp de concentration. Rien n'est plus éloigné du conte mais cette interprétation de la 
métamorphose est caractéristique des intentions de Fühmann. À ses yeux le conte est figé mais le mythe continue à vivre. Pour un spectateur moderne la malédiction ne consiste pas à être transformé en animal mais à perdre d'une autre façon son humanité et les images des hommes dégradés par la détention dans les camps de concentration hantent les esprits de la seconde moitié du XX $\mathrm{XX}^{\mathrm{e}}$ siècle ${ }^{18}$. Dans le conte la métamorphose est réversible, ainsi dans Les sept corbeaux des frères Grimm, mais chez Fühmann, au moment du départ d'Ulysse, la nymphe Cassiphoné, qui a manifesté son amour pour un Grec, est transformée par Circé en lémure. Le cycle de la malédiction n'est pas interrompu.

9 Fühmann introduit une seconde modification importante. Elle porte sur l'intervention divine qui permet à Ulysse d'échapper aux maléfices de Circé. Fühmann transforme le motif de conte qu'est l'affrontement du héros et de la magicienne, après qu'un être surnaturel a armé le héros d'un auxiliaire magique. Chez Homère, c'est le dieu Hermès qui donne à Ulysse, habituellement protégé par Athéna, la plante, le moly, qui lui permet d'échapper aux effets de la baguette magique de Circé. Fühmann modifie délibérément la nature de l'affrontement. Il explique qu'il considère comme plus intéressant de faire lutter deux femmes et il remplace l'intervention d'Hermès par celle d'Athéna. C'est donc Athéna qui s'oppose à Circé, lorsqu'Ulysse rencontre la magicienne. Pourquoi ce changement ? Fühmann considère Athéna comme la déesse du patriarcat, une déesse virile, et Circé comme la déesse du matriarcat, déesse de la vie et de la régénération, symbolisée par la toile d'or qu'elle file et qui l'accompagne tout au long du ballet comme son attribut. Ce faisant, Fühmann s'éloigne de l'intemporalité du conte pour actualiser la rencontre d'Ulysse et de Circé, car le débat sur le patriarcat et le matriarcat agite les esprits au début des années 1980. Il est par exemple au centre du livre de Christa Wolf Kassandra, dans lequel toute la violence est attribuée au triomphe des valeurs du patriarcat, tandis qu'est évoqué en contrepoint le monde paisible et créateur du matriarcat, sous forme de la vie des femmes dans les grottes près de Troie. Une vision mythique de l'histoire s'impose qui fait succéder au matriarcat, période de paix, le patriarcat, longue suite de conflits dans laquelle s'inscrit le début des années 1980, moment de tensions internationales, où la querelle des missiles fait craindre une troisième guerre mondiale, le cataclysme général. «Le mythe renvoie expressément à la réalité » («Der Mythos verweist nachdrücklich auf die Realität» $\left.{ }^{19}\right)$. Conformément au pessimisme ambiant c'est Athéna, symbole du patriarcat, qui l'emporte sur Circé, déesse du matriarcat.

Le troisième point où s'exprime l'originalité de Fühmann, est la séparation de Circé et d'Ulysse. Ce qui nous frappe chez Homère, qu'il s'agisse de la séparation de la nymphe Calypso et d'Ulysse ou de la séparation de Circé et d'Ulysse, c'est l'absence de toute note tragique. Calypso aide Ulysse à préparer son bateau, elle lui donne nourritures et vêtements avant son départ. Circé prodigue à Ulysse ses conseils pour qu'il évite Charybde et Scylla sur le chemin du retour vers Ithaque (chant XII). Cette absence de passion semble être celle des personnages des contes, dont Fühmann considère qu'ils sont des «ombres", alors que les mythes nous présentent des êtres dans leur réalité, réalité qu'il actualise là également («Im Mythos sind Menschen wie Götter ganze, unverkrüppelte Wesen. Im Märchen haben die Wesen nur Schattencharakter $\left.{ }^{20}\right)$. Dans son ballet il traite Circé, à l'instar de Calderon dans El mayor encanto amor (1635), plus comme une Didon abandonnée que comme une femme détachée. Le début des années 1980 coïncide avec la crête de la vague féministe. Des organisations de femmes sont créées. De multiples livres féministes paraissent en R.F.A., en R.D.A. comme en France. 
Il suffit de citer encore une fois la Kassandra de Christa Wolf, l'œuvre militante de Christine Brückner Wenn du geredet hättest, Desdemona, en France le livre de Catherine Clément, L'Opéra ou la défaite des femmes. Dans tous ces ouvrages la femme est présentée comme une victime. Les héros masculins sont démasqués et apparaissent comme des brutes, ainsi Achille que Cassandre appelle «das Vieh», ou comme des égoïstes qui oppriment leurs compagnes, ainsi les grands hommes chez Christine Brückner, tandis que Catherine Clément montre que les héroïnes d'opéra sont toutes des femmes sacrifiées. Fühmann présente Circé à la fin de son ballet comme une femme délaissée et furieuse de l'être, qui reste vouée à la solitude de son île, réduite à la compagnie des nymphes et des lémures. De rage elle arrache la toile d'or qui l'enveloppe, la déchire et en éparpille les morceaux dans son palais au moment où Ulysse repart dans son bateau.

11 Ulysse n'est pas présenté pour autant sous des traits négatifs. Or la tradition aurait pu y inviter. Euripide par exemple, dans Iphigénie en Aulide, fait de lui un personnage peu sûr et pendant toute une partie du Moyen Age les écrivains insistent sur sa duplicité et font même de lui un meurtrier ${ }^{21}$. Homère avait complètement gommé ces aspects de la personnalité d'Ulysse. Fühmann lui attribue devant Circé des sentiments complexes : il est séduit par la beauté de la magicienne, mais elle lui inspire en même temps de la haine, en raison du traitement qu'elle a fait subir à ses compagnons. Fidèle à Ithaque et à Pénélope, il n'oublie pas son but devant Circé et lui demande de repartir, mais il souhaiterait une séparation en bons termes et tend la main à Circé au moment où il la quitte (p. 50). Circé refuse de saisir cette main. Alors que chez Homère l'épisode de Circé se termine par le départ pour Ithaque, chez Fühmann le ballet finit dans les ténèbres. Un autre épisode illustre ce qu'on pourrait appeler un glissement vers le tragique : chez Homère le compagnon d'Ulysse, Elpénor, se tue accidentellement parce qu'il a trop bu, chez Fühmann il est désigné par tirage au sort comme le seul Grec devant rester sur l'île de Circé et choisit le suicide. Le mythe ignore le happy end qui caractérise le conte («Der Mythos kennt kein Happy-End» ${ }^{22}$ ).

Une autre forme d'opposition entre le mythe et le conte réside dans le fait que le conte se déroule en surface alors que le mythe se déploie dans l'espace («Märchen sind Linien auf der Fläche, Mythen Bewegungen im Raum» ${ }^{23}$ ). Le ballet est donc une forme d'expression qui convient tout particulièrement au mythe, qui se prête à la représentation plastique (on le trouve par exemple sur les vases grecs, Titien avec Marsyas et Rembrandt avec Danaé le choisissent comme sujet de tableau ${ }^{24}$ ). Le ballet met le mythe en mouvement, souligne son caractère vivant grâce à ses moyens propres : la lumière, la musique, la chorégraphie. Là encore Fühmann évite la féerie du conte pour insister sur les dissonances du mythe.

13 La scène est divisée en trois parties : à la partie supérieure se trouve le palais de Circé, le plus souvent baigné de clarté, tandis que les lémures sont à l'étage inférieur et œuvrant dans l'ombre, ils ne sont éclairés qu'au moment où la danse réunit Circé, Ulysse, les nymphes, les Grecs et les lémures, dans un moment d'allégresse éphémère, une fête de la réconciliation telle que la rêve Hölderlin. Cet étagement souligne la division de la société en classes. Mais Fühmann utilise d'autres procédés que la disposition scénique pour souligner les antagonismes, la gestuelle par exemple. Lorsque les Grecs retrouvent leur forme humaine, leurs attitudes illustrent le processus du passage d'un état à un autre qu'Ovide a décrit dans ses détails. Des bêtes de somme retrouvent leur dignité d'homme. De même la rencontre d'Athéna et de Circé est représentée sur la scène plutôt comme le heurt des deux principes du patriarcat et du 
matriarcat que comme la rencontre de deux déesses. À l'approche d'Athéna la toile d'or qui environne Circé s'efface. Athéna est entourée d'une lumière bleue, plus dure, plus froide que la lumière dorée qui est celle de Circé. Lorsqu'Athéna triomphe de Circé la lumière bleue se répand sur la scène, symbolisant la victoire du principe de dureté.

Enfin la danse exprime l'affrontement de l'homme et de la femme dans le couple d'Ulysse et de Circé. Au moment de la fête générale tous se joignent à cette danse dans la musique et la lumière, puis les nymphes et les Grecs se séparent, préfigurant la séparation d'Ulysse et de Circé. Les dernières figures du ballet se déroulent dans un climat de désolation et le tout s'achève dans les ténèbres. Le palais de Circé n'est plus illuminé que par des éclairs. La nuit tombe, la tempête se déclare. Circé ôte ses vêtements, une musique barbare retentit et Fühmann conclut : « Il n'est pas nécessaire que le rideau retombe. Il fait nuit noire » (p. 52).

Le ballet de Fühmann, faute de musique, n'a jamais été représenté, mais le texte a été publié en même temps qu'une série de dessins exécutés par Wieland Förster, à qui Fühmann a demandé en vain, à plusieurs reprises d'illustrer ses ouvrages. Un premier projet pour le Marsyas n'avait pas abouti parce que Förster répugne à se faire illustrateur, dans la mesure où son art fuit l'anecdote et le décoratif et où il estime qu'un bon texte se suffit à lui-même. Mais Förster et Fühmann ont évoqué ensemble l'intérêt du personnage de Circé au cours d'un entretien radiophonique avec Luise Köpp en $1975^{25}$, dans la mesure où cette femme est le lieu de contradictions, symbole de séduction et de danger. Quelques années plus tard, au moment où la maison d'édition Hinstorff se propose de publier l'argument du ballet Kirke und Odysseus, Wieland Förster a terminé une série de dessins "Couples d'amants» (Liebespaare). Les deux artistes décident de présenter le texte et les dessins parallèlement, de faire œuvre commune en témoignage d'amitié. Ce sont, précise Förster dans un entretien avec Peter Liebers après la mort de Fühmann, deux productions indépendantes qui viennent se compléter et peut-être s'enrichir. Il ajoute qu'elles ont le même sujet: l'amour ${ }^{26}$. Les amants de Förster apparaissent comme des blocs de rochers aux formes humaines qui s'affrontent, se confondent, se séparent. Le dernier dessin représente une femme qui veille encore près d'une masse repliée sur elle-même sur un ciel sombre. Ces dessins participent du mythe, tel que le définit Fühmann, puisqu'ils montrent l'amour dans sa force élémentaire ${ }^{27}$ et dans ses contradictions, obéissant à la dialectique du rapprochement et de l'éloignement. Cette série de dessins a été réalisée en même temps qu'un autre cycle, très sombre, intitulé Labyrinthe, exprimant la menace de destruction de la terre par une guerre atomique ${ }^{28}$. Les couples d'amants constituent le contrepoint du labyrinthe ${ }^{29}$ : ils sont l'espoir, la vie. Il existe entre le labyrinthe et les couples d'amants un rapport de polarité analogue à celui qui lie le texte de Fühmann et les dessins de Förster.

Considérant que le conte et le mythe sont au cœur de la littérature, Fühmann n'a cessé de méditer sur les deux formes de récit et de les pratiquer dans son œuvre. Il a, au fil des années, comme il l'a fait ici peu de temps avant sa mort, privilégié le mythe. S'il a d'abord pensé que les contes deviendraient réalité dans la nouvelle société socialiste ${ }^{30}$, il a par la suite exprimé sa préférence pour le mythe qui seul rend compte des 
contradictions qui caractérisent la réalité ${ }^{31}$, alors que le conte relève du rêve («Wunschdenken» ${ }^{32}$ ).

La dépréciation du conte au profit du mythe implique de surcroît une intention polémique : en prônant la supériorité du mythe, Fühmann dénonce les faiblesses de certaines productions littéraires de la R.D.A. dont le prétendu réalisme relève plutôt du conte, $\mathrm{du}$ «Wunschdenken», dans la mesure où elles ne présentent que des contradictions susceptibles de trouver une solution et s'exposent au danger de simplifier et de moraliser la réalité ${ }^{33}$. Fühmann ne dit-il pas : "Celui qui cherche dans l'art la confirmation de ses illusions, est un ennemi de l'art, même s'il prétend que l'art est sacré $~^{34}$. Le retour au mythe signifie aussi chez Fühmann l'exigence d'un art de qualité, d'un art problématique, utilisant la tradition, un art qu'il a défendu en R.D.A. en se faisant découvreur de talents, au besoin contre l'art officiel et établi. Christa Wolf note après sa mort: "son souci, sa préoccupation étaient de savoir s'il y aurait une nouvelle génération de poètes dans ce pays, s'il y aurait une littérature digne de ce nom $»^{35}$. Pour Fühmann, au cœur de cette littérature, les mythes continueraient à vivre $^{36}$, puisque les vrais poètes ne se lassent pas de les raconter.

\section{NOTES}

1. - Franz Fühmann, Ein Ballett, Kirke und Odysseus. Liebespaare. Ein graphischer Zyklus von Wieland Förster. Rostock, 1984. Les chiffres entre parenthèses indiquent les pages de cette édition.

2. - Elisabeth Frenzel, Stoffe der Weltliteratur, Stuttgart, 1970, article Odysseus, p. 548 ss.

3. - Franz Fühmann, Prometheus. Die Titanenschlacht, Berlin, D.D.R., 1974.

4. - Gero von Wilpert, Sach wörterbuch der Literatur, Stuttgart, 1969, p. 463 et 505.

5. - Franz Fühmann, 22 Tage oder die Hälfte des Lebens, Rostock, 1975.

6. - Franz Fühmann, Erfahrungen und Widersprüche, Versuche über Literatur, Rostock, 1975. Das mythische Element in der Literatur, p. 147 à 219.

7. - Lettre inédite de Wieland Förster à l'auteur de l'article, datée du 6.2.1991.

8. - Sinn und Form, 1985, Heft 2, p. 297.

9. - Franz Fühmann, Die Richtung der Märchen, Gedichte, Berlin, 1962.

10. -22 Tage, p. 217 («Trümmer von Mythen») et p. 219 ss.

11. - Erfahrungen und Widersprüche, p. 157.

12. - Vladimir Propp, La morphologie du conte, Paris, 1970.

13. - Erfahrungen und Widersprüche, p. 157.

14. - 22 Tage, p. 225 : vom Märchen zum Mythos heißt: zum vollen Leben, zumganzen Menschen, zur dialektischen Realität».

15. -22 Tage, p. 182.

16. - Ovide, Les Métamorphoses, XIV, v. 257 à 328.

17. - Apollonios de Rhodes, Les Argonautiques, ch. IV, v. 672 ss.

18. - 22 Tage, p. 205 : «Du kannst tun, was du willst, du kommst von Auschwitznicht mehr los». Wieland Förster partage cette obsession cf. son journal de voyage en Tunisie Begegnungen, Berlin, 1977, p. 26. 
19. - Erfahrungen und Widersprüche, p. 167.

20. - 22 Tage, p. 220.

21. - Elisabeth Frenzel, Stoffe der Weltliteratur, p. 550.

22. - Erfahrungen und Widersprüche, p. 196.

23. - 22 Tage, p. 221.

24. - Erfahrungen und Widersprüche, p. 175.

25. - Wieland Förster, Einblicke, Berlin DDR, 1985, p. 55.

26. - Sinn und Form, 1985, Heft 2, p. 297.

27. - Cf. 22 Tage, p. 226 : «oft sind Märchen Juwelen, die Mythen nur Rohdiamanten».

28. - W. Förster, Einblicke, p. 136.

29. - W. Förster, Labyrinth, Berlin DDR, 1988, p. 138 : «Angefangen, Liebespaare zu zeichnen - sie sind das Gegengewicht zum Labyrinth».

30. - Horst Lohr, Vom Märchen zum Mythos. Zum Werk Franz Fühmanns. in Weimarer Beiträge, I, 1982, p. 62 ss. : «die Märchen werden Wirklichkeit werden».

31. - Erfahrungen und Widersprüche, p. 162: «Der Mythos gibt den Widerspruch wieder, das Märchen aber schafft ihn weg».

32. - Erfahrungen und Widersprüche, p. 196. «Der Mythos kennt... kein Wunschdenken».

33. - 22 Tage, p. 220 : «Dennoch sind noch im Märchen bestimmende Züge des Lebens sichtbar, zum Unterschied von mancher Literatur, die sich realistisch nennt und nur Papier ist».

34. - Erfahrungen und Widersprüche, p. 196: «Wer in der Kunst nur seine Illusionen bestätigt finden will, der will im Grunde genommen Kunst gar nicht, auch wenn er beteuert, sie sei ihm heilig».

35. - Sinn und Form, 1984, 5. Heft, p. 1021 : «Ob in diesem Land Dichter nachwuchsen; ob es eine Literatur geben wird, die diesen Namen verdient - das war seine ureigene Sorge und Bekümmernis».

36. - 22 Tage, p. 223. «Mythen... ereignen sich ununterbrochen, und wer sie sieht und erzählen kann, ist ein Dichter».

\section{RÉSUMÉS}

Les notions de conte et de mythe sont au centre de l'œuvre de Franz Fühmann et de sa réflexion sur la littérature. Il les a définies en les opposant dans son journal de voyage en Hongrie 22 Tage oder die Hälfte des Lebens sous forme d'aphorismes et dans une conférence intitulée Das mythische Element in der Literatur. En étudiant plus particulièrement son adaptation, dans un argument de ballet, de l'histoire d'Ulysse et de Circé, nous voulons montrer à l'aide de trois exemples comment il va du conte au mythe, c'est-à-dire comment il s'éloigne du merveilleux pour se rapprocher de la réalité de son temps. Les compagnons d'Ulysse ne sont pas transformés en pourceaux mais en êtres déshumanisés qui évoquent un univers concentrationnaire. L'affrontement du héros, Ulysse, aidé par Athéna, et de la magicienne Circé devient une lutte entre déesses symbolisant le patriarcat et le matriarcat, car le triomphe du principe du patriarcat sur celui du matriarcat est souvent considéré comme une clé de l'histoire de l'humanité au début des années 80. Ces années se caractérisent également par une vague de féminisme dont nous trouvons l'écho dans la troisième modification introduite par Fühmann : la séparation de Circé et d'Ulysse, plus tragique que chez Homère. 
Le texte a été publié en même temps qu'une série de dessins de Wieland Förster, sculpteur, ami de Fühmann. Il ne s'agit pas d'illustrations véritables mais plutôt d'œuvres croisées, dans un rapport dialectique, autour d'un même thème : le couple d'amants.

En conclusion nous suggérons que Fühmann oppose conte et mythe comme il oppose la littérature du «Wunschdenken» et du Happy end et la littérature de qualité qui ne cesse d'explorer la réalité riche en contradictions, la réalité des grands mythes.

Die beiden Begriffe Märchen und Mythos stehen im Mittelpunkt des Werkes Franz Fühmanns und seiner Reflexion über Literatur. Er hat sie dadurch definiert, daß er sie gegenüberstellte, sei es in seinem Reisetagebuch aus Ungarn oder in einem Vortrag, den er Das mythische Element in der Literatur betitelt hat. Ich will seine Bearbeitung der Geschichte von Odysseus und Kirke in einem Ballett untersuchen und anhand von drei Beispielen zeigen, wie er vom Märchen zum Mythos übergeht, das heißt wie er sich vom Wunderbaren entfernt, um sich der Wirklichkeit seiner Zeit anzunähern.

Die Gefährten des Odysseus werden nicht in Schweine, sondern in Wesen verwandelt, die ihre menschliche Würde eingebüßt haben und an die Häftlinge eines Konzentrationslagers mahnen. Die Konfrontation des Helden Odysseus, der von Athena unterstützt wird, und der Zauberin Kirke wird zu einem Kampf zwischen zwei Göttinnen, die das Patriarchat und das Matriarchat versinnbildlichen, denn der Triumph des Patriarchats über das Matriarchat wird in den frühen 80er Jahren oft als Schlüssel gebraucht zum Verständnis der Geschichte der Menschheit. Diese Jahre werden zugleich vom Feminismus geprägt, dessen Wirkung wir in der dritten von Fühmann erarbeiteten Veränderung finden, nämlich die der Trennung von Odysseus und Kirke, die ganz anders als bei Homer eine Wendung ins Tragische erfährt.

Der Text ist zugleich mit einem graphischen Zyklus von Wieland Förster, einem Bildhauer, der mit Fühmann eng befreundet war, veröffentlicht worden. Es handelt sich nicht um Illustrationen im üblichen Sinne, sondern eher um aufeinander dialektisch bezogene Werke, die das gleiche Thema beschreiben, das Liebespaar.

Abschließend möchte ich andeuten, daß Fühmann das Märchen dem Mythos entgegensetzt, wie er einer Literatur des Wunschdenkens und des Happy-End eine Literatur mit Niveau gegenüberstellt, die ununterbrochen eine an Widersprüchen reiche Realität durchforscht und befragt, die Realität der großen Mythen.

\section{AUTEUR}

\section{GENEVIÈVE CIMAZ-MARTINEAU}

Université de Paris IV 